\title{
Imaging mass spectrometry reveals heterogeneity of proliferation and metabolism in atherosclerosis
}

\author{
Christelle Guillermier, ${ }^{1,2,3}$ Sean P. Doherty, ${ }^{4}$ Adam C. Whitney, ${ }^{2,3}$ Vladimir R. Babaev, ${ }^{4}$ \\ MacRae F. Linton, ${ }^{4}$ Matthew L. Steinhauser, ${ }^{1,2,3,5}$ and Jonathan D. Brown ${ }^{4}$ \\ ${ }^{1}$ Harvard Medical School, Boston, Massachusetts, USA. ${ }^{2}$ Center for Nanolmaging and ${ }^{3}$ Department of Medicine, Division of \\ Genetics, Brigham and Women's Hospital, Boston, Massachusetts, USA. ${ }^{4}$ Division of Cardiovascular Medicine, Vanderbilt \\ University Medical Center, Nashville, Tennessee, USA. ${ }^{5}$ Department of Medicine, Division of Cardiovascular Medicine, \\ Brigham and Women's Hospital, Boston, Massachusetts, USA.
}

\begin{abstract}
Atherosclerotic plaques feature local proliferation of leukocytes and vascular smooth muscle cells (VSMCs) and changes in cellular metabolism. Yet the relationship between glucose utilization and proliferation has been technically impossible to study directly in cells of atherosclerotic plaques in vivo. We used multi-isotope imaging mass spectrometry (MIMS), a quantitative imaging platform, to measure coincident cell division and glucose utilization at suborganelle resolution in atherosclerotic plaques. In established plaques, $65 \%$ of intimal foam cells and only $4 \%$ of medial VSMCs were labeled with ${ }^{15} \mathrm{~N}$-thymidine after 1 week of isotope treatment. Dividing cells demonstrated heightened glucose labeling. MIMS detected ${ }^{2} \mathrm{H}$-glucose label in multiple subcellular compartments within foam cells, including lipid droplets, the cytosol, and chromatin. Unexpectedly, we identified an intensely focal region of ${ }^{2} \mathrm{H}$-label in VSMCs underlying plaques. This signal diminished in regions of aorta without atherosclerosis. In advanced plaques, ${ }^{15} \mathrm{~N}$-thymidine and ${ }^{2} \mathrm{H}$-glucose labeling in foam cells and VSMCs significantly decreased. These data demonstrate marked heterogeneity in VSMC glucose metabolism that was dependent on both proliferative status and proximity of VSMCs to plaques. Furthermore, these results reveal how quantitative mass spectrometry coupled with isotope imaging can complement other methods used to study cell biology directly in the growing atherosclerotic plaque in vivo.
\end{abstract}

Authorship note: MLS and JDB are co-senior authors.

Conflict of interest: The authors have declared no conflict of interest exists.

Copyright: (c) 2019 American Society for Clinical Investigation

Submitted: March 5, 2019

Accepted: April 25, 2019

Published: June 6, 2019.

Reference information: /Cl Insight. 2019;4(11):e128528. https://doi. org/10.1172/jci.insight.128528

\section{Introduction}

The growth of atherosclerotic plaques occurs through recruitment and local proliferation of macrophages and vascular smooth muscle cells (VSMCs) and their maturation to lipid-laden foam cells (1). Foam cells, activated macrophages, and VSMCs feature significant changes in cell metabolism, including augmented increased glucose uptake, glycolysis, lactate production, and reduced oxidative phosphorylation measured in vitro (2-4). These cell type-specific changes in metabolism may reflect pathologic metabolic reprogramming, akin to the Warburg effect described in rapidly growing tumors (2). Despite the potential significance of these biological processes in atherosclerosis, the functional links between proliferation and metabolism in different cell types remain poorly understood in vivo, in part because they are technically challenging to study simultaneously directly in plaque. Thus, new methods capable of evaluating the functional heterogeneity of cells in their endogenous biologic context within the vessel wall are needed.

The strong connections among inflammation, cell growth, and metabolism provided the rationale for us to quantify proliferation and glucose utilization simultaneously in experimental atherosclerosis using multi-isotope imaging mass spectrometry (MIMS). MIMS merges stable isotope tracer methodology with nanoscale secondary ion mass spectrometry, thereby enabling multiplexed quantification of stable isotope tracers at suborganelle resolution (5-8). We administered tracers for cellular proliferation $\left({ }^{15} \mathrm{~N}\right.$-thymidine) and glucose utilization ( ${ }^{2} \mathrm{H}$-glucose) to individual $\mathrm{Ldll}^{-/}$animals after 8 weeks and 21 weeks on an atherogenic diet and quantitatively mapped tracer incorporation in aortic plaques. We discovered a correlation between glucose utilization and cellular proliferation in early plaques that was particularly robust for intimal foam cells. Unexpectedly, a gradient of glucose signal was evident in VSMCs directly underlying lesions despite lower rates of proliferative 
activity in these cells. These data implicate focal changes in VSMC metabolism as a feature of VSMC phenotypic plasticity in atherosclerosis. In advanced plaques, the tracer signals were diminished, suggesting proliferative exhaustion and/or loss of activity of factors that drive these processes in the plaque.

\section{Results}

MIMS maps proliferation and glucose utilization by key cell types within the complex architecture of the atherosclerotic plaque. To establish atherosclerotic plaques, we fed $L l d r^{-1}$ mice a high-fat and high-cholesterol (HF/ $\mathrm{HC}$ ) diet for 9 weeks. We administered ${ }^{2} \mathrm{H}$-glucose during the final 3 days to map glucose utilization within plaques. In order to test for preferential glucose utilization by a proliferating subpopulation of cells, we administered ${ }^{15} \mathrm{~N}$-thymidine, a stable isotope tracer of DNA synthesis and cell division, for 7 days prior to sacrifice (Figure 1A). At the end of the labeling period, mice were euthanized and aortic roots were fixed, embedded, and sectioned for MIMS analysis. Staining of aortic root sections using toluidine blue demonstrated atherosclerotic lesions in the aortic sinuses, with lipid appearing dark gray due to reactivity with osmium tetroxide (Figure 1A). We used these stained sections to identify characteristic aortic plaques and define target areas for MIMS analyses, which were performed on adjacent sections. Prior work demonstrated that imaging of ${ }^{12} \mathrm{C}^{14} \mathrm{~N}^{-}$and ${ }^{31} \mathrm{P}^{-}$ions can capture cell type-specific features in complex tissue sections $(5,9,10)$. Indeed, ${ }^{12} \mathrm{C}^{14} \mathrm{~N}^{-}$and ${ }^{31} \mathrm{P}^{-}$imaging defined overall plaque architecture, detailing major cellular constituents within plaques based on location in the vessel wall and characteristic intracellular features (Figure 1A). Vascular endothelial cells (ECs) - positioned at the interface between aortic lumen and underlying atheroma - were flat, elongated, and oriented longitudinally (Figure 1B). Foam cells appeared as a honeycombed lattice containing numerous cytoplasmic lipid droplets (Figure 1, A and B). Finally, medial VSMCs were cuboidal/columnar cells subjacent to intimal foam cells and bounded by undulating bands of elastin, characterized by intense ${ }^{12} \mathrm{C}^{14} \mathrm{~N}^{-}$signal (Figure 1, A and C). Simultaneous imaging of ${ }^{31} \mathrm{P}^{-}$ions also defined nuclei due to the high phosphorus content in DNA and histone proteins, as previously described (Figure 1B and Supplemental Figure 1; supplemental material available online with this article; https://doi.org/10.1172/jci.insight.128528DS1) (11, 12).

In addition to ${ }^{12} \mathrm{C}^{14} \mathrm{~N}^{-}$and ${ }^{31} \mathrm{P}^{-}$imaging for histological identification, MIMS quantitatively maps the incorporation of up to 4 stable isotope tracers through measurement of the corresponding isotope ratios: an increase in the isotope ratio above the natural ratio indicates tracer incorporation (13). As we have previously demonstrated, ${ }^{15} \mathrm{~N}$-thymidine labeling — reflecting DNA synthesis within the 7-day labeling period - was detected in the nuclei of a subset of cells in all layers of the vessel wall (Figure 1, B and C) $(5,9$, 10). In each cell type within the plaques, strong ${ }^{2} \mathrm{H}^{-}$signal above background levels, indicative of ${ }^{2} \mathrm{H}$-glucose labeling, was also readily detected. Collectively, these data demonstrate the utility of MIMS for multiplexed measurements of stable isotope tracers in atherosclerotic plaque at suborganelle imaging resolution.

Subcellular glucose metabolism is enriched in proliferating foam cells. We next applied MIMS to study the relationship between glucose metabolism and proliferation in cells within established atherosclerotic lesions. We first focused on the vascular intima. Both ECs and foam cell nuclei were labeled with ${ }^{15} \mathrm{~N}$-thymidine (Figure $1 \mathrm{~A}$ and Figure 2A). While the total number of detectable ECs for mapping thymidine was low ( $n=10$ counted from 3 mice), the rate of ${ }^{15} \mathrm{~N}$-labeling averaged $17 \%$ (data not shown). The total number of foam cell nuclei visible in the imaging plane was higher ( $n=62$ nuclei). Overall, $65 \%$ of the foam cell nuclei were labeled with ${ }^{15} \mathrm{~N}$-thymidine, revealing a high rate of DNA synthesis in these cells over the 1-week labeling period (Table 1). Notably, one foam cell contained a second nucleus with markedly higher ${ }^{15} \mathrm{~N}$-signal (Figure $2 \mathrm{~A}$ ). This nucleus could represent DNA from a phagocytosed, apoptotic cell, but is unlikely to be a telophase nucleus, given the extremely skewed ${ }^{15} \mathrm{~N}$-signal. Simultaneous measurements of ${ }^{2} \mathrm{H}$-glucose labeling identified overall similar trends in ${ }^{2} \mathrm{H}$-signal in both the EC and foam cell populations (Figure $2 \mathrm{~B}$ ). The low EC numbers do limit statistical power for direct comparisons. Due to the large size of foam cell cytoplasm relative to their nucleus, only a subset of foam cells in any given plane of imaging contained a visible nucleus. Therefore, we narrowed our analysis to include only those foam cells in which the imaging plane captured the nucleus and compared the distribution of ${ }^{2} \mathrm{H}$-glucose labeling as a function of concomitant ${ }^{15} \mathrm{~N}$-thymidine labeling. With this approach, we identified a statistically significant increase in ${ }^{2} \mathrm{H}$-glucose labeling in foam cells that had recently divided $\left({ }^{15} \mathrm{~N}^{+}\right)$(Figure 2C). In dividing cells, glucose consumption supports biomass production via the activity of multiple metabolic pathways located in the cytoplasm and nucleus. We hypothesized that ${ }^{2} \mathrm{H}$-glucose label would distribute nonuniformly in organelles of individual cells within the plaque. We quantified ${ }^{2} \mathrm{H}$ enrichment in subcellular structures within individual foam cells. Among ${ }^{15} \mathrm{~N}$-thymidine-positive foam cells, we 
A
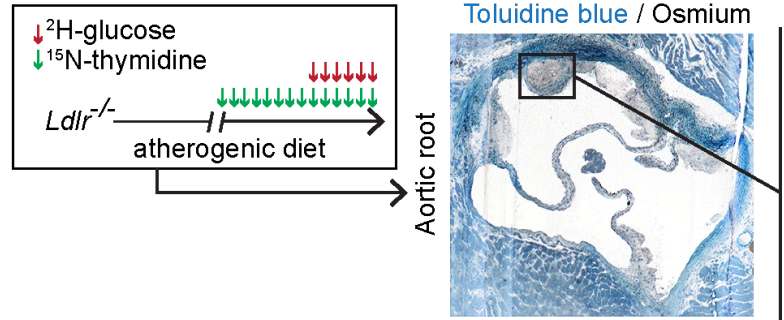

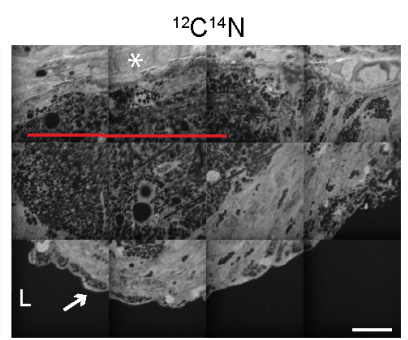

B

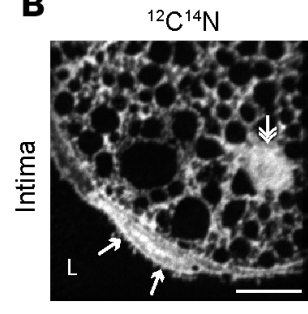

C

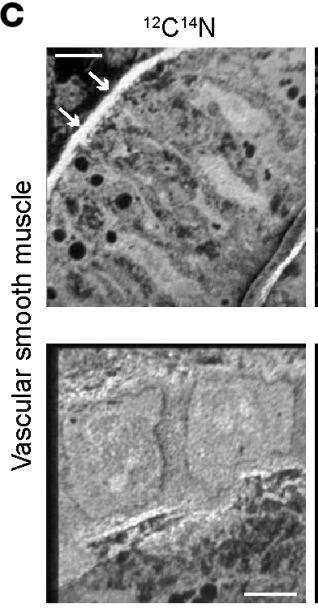

${ }^{31} \mathrm{P}$

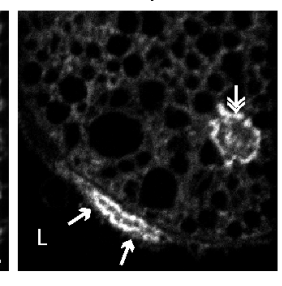

${ }^{31} \mathrm{P}$
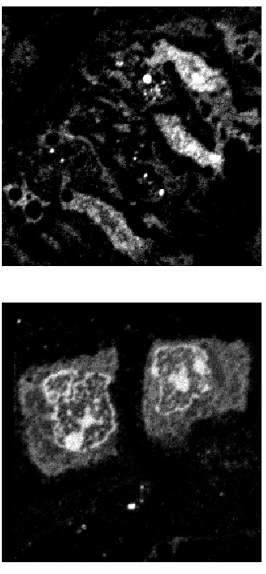

${ }^{15} \mathrm{~N} /{ }^{14} \mathrm{~N}\left({ }^{15} \mathrm{~N}\right.$-thymidine $){ }^{2} \mathrm{H} /{ }^{1} \mathrm{H}\left({ }^{2} \mathrm{H}\right.$-glucose $)$
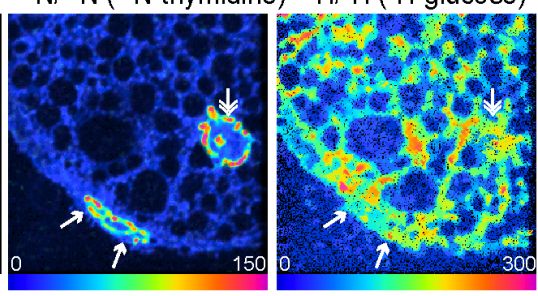

${ }^{15} \mathrm{~N} /{ }^{14} \mathrm{~N}\left({ }^{15} \mathrm{~N}\right.$-thymidine $) \quad{ }^{2} \mathrm{H} /{ }^{1} \mathrm{H}\left({ }^{2} \mathrm{H}\right.$-glucose $)$
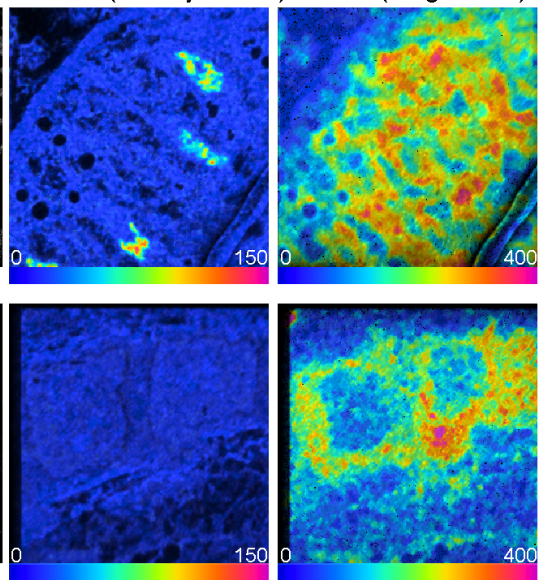

Figure 1. Multi-isotope imaging mass spectrometry maps proliferation and glucose utilization by key cell types within the complex architecture of atherosclerotic plaque. (A) Schematic depicting the application of multi-isotope imaging mass spectrometry (MIMS) to atherosclerosis (left). Ldlr/- mice were fed a high-fat/high-cholesterol diet. Stable isotope labeling by intraperitoneal injections of ${ }^{15} \mathrm{~N}$-thymidine ( 1 week) and ${ }^{2} \mathrm{H}$-glucose ( 3 days) was initiated before sacrifice. The fixation protocol included osmium tetroxide to preserve lipids (black stain). Toluidine blue staining (middle) revealed atherosclerotic plaques in the aortic roots and guided coordinate selection for MIMS analysis (right). The image shown represents quantification of ${ }^{12} \mathrm{C}^{14} \mathrm{~N} \mathrm{~N}^{-}$and provides histological detail. White arrow, endothelial cell (EC); top white asterisk, VSMC; red line, Foam cell core; L, lumen. Scale bar: $20 \mu \mathrm{m}$. (B) Quantitative mass images reveal features of cells in atherosclerotic plaque intima: EC (white arrows) and numerous foam cells with lipid droplets (dark spaces) in ${ }^{12} \mathrm{C}^{14} \mathrm{~N}^{-}$images. Phosphorus ( $\left.{ }^{31} \mathrm{P}^{-}\right)$images reveal nuclei due to high phosphorus content of chromatin, including foam cell nucleus (2-headed arrow). Hue saturation intensity images are used to display the isotope ratio measurements and map the incorporation of ${ }^{15} \mathrm{~N}$-thymidine $\left({ }^{12} \mathrm{C}^{15} \mathrm{~N}^{-} /{ }^{12} \mathrm{C}^{14} \mathrm{~N}^{-}\right)$and ${ }^{2} \mathrm{H}$-glucose $\left({ }^{12} \mathrm{C}_{2}{ }^{2} \mathrm{H} /{ }^{12} \mathrm{C}_{2}{ }^{1} \mathrm{H}\right)$. The lower bound of the scale is set to the background ratio $(0 \%)$, and the upper bound is set to reveal differences in labeling ( $150 \%$ and $300 \%$ above background, respectively). Scale bar: $5 \mu \mathrm{m}$. (C) MIMS images of vascular smooth muscle cells (VSMCs) underlying atherosclerotic plaques. Elastin bands (white arrows) appear bright in ${ }^{12} \mathrm{C}^{14} \mathrm{~N}^{-}$images. Scale bar: $5 \mu \mathrm{m}$.

detected a modest increase in ${ }^{2} \mathrm{Hsignal}$ in the lipid droplets over background levels, with more substantial elevations in the cytosolic and chromatin fractions of the cells (Figure 2D). The outlier measurements in chromatin corresponded to the glucose signal from the second nucleus in the foam cell in Figure 2A. Collectively, these data demonstrate the following: (i) more than half of foam cells in established lesions divided within the preceding 1-week period; (ii) dividing foam cells utilized glucose at a higher rate than nondividing cells; and (iii) the metabolic fate of glucose was nonuniform, with both cytosolic and nuclear compartments contributing significantly to the overall glucose signals within intimal cells. 
A
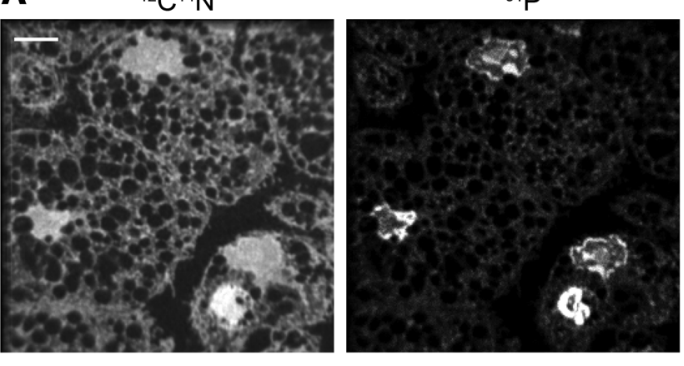

${ }^{15} \mathrm{~N} /{ }^{14} \mathrm{~N}\left({ }^{15} \mathrm{~N}\right.$-thymidine $)$

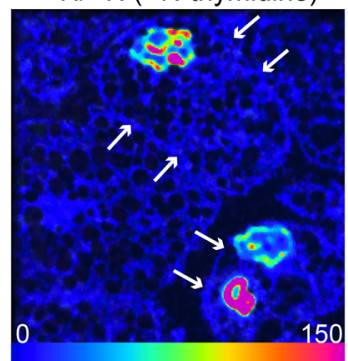

${ }^{2} \mathrm{H} /{ }^{1} \mathrm{H}\left({ }^{2} \mathrm{H}\right.$-glucose $)$

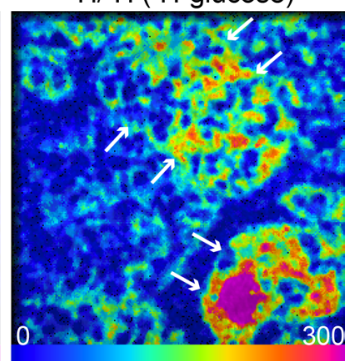

B

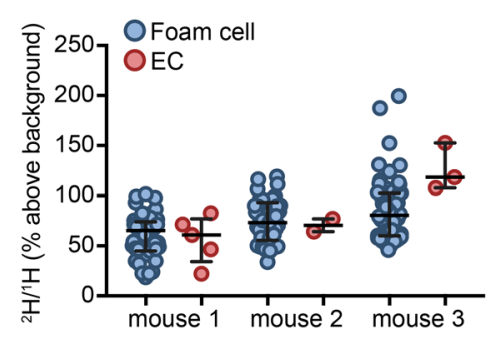

c

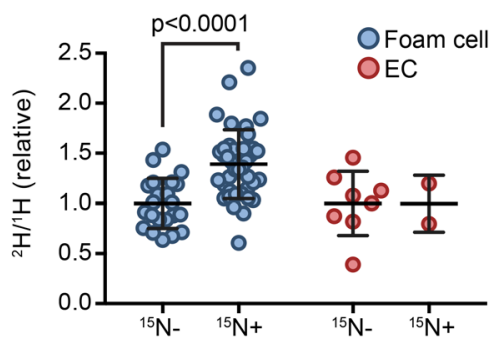

D

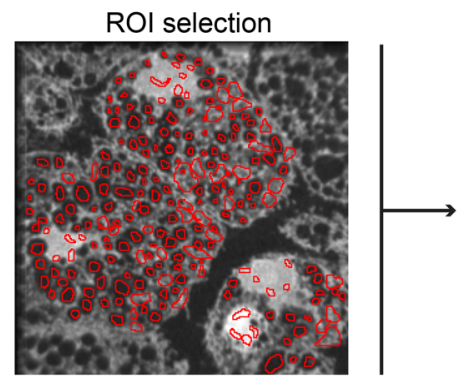

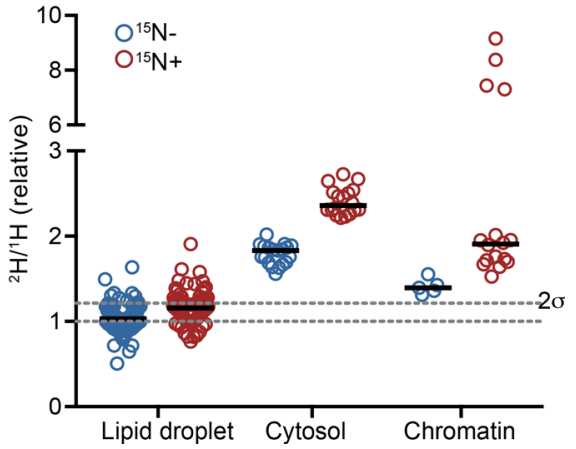

Figure 2. Subcellular glucose utilization is enriched in proliferating foam cells. (A) Intimal foam cells. These images reveal higher-intensity ${ }^{2} \mathrm{H}$-glucose labeling in divided ${ }^{15} \mathrm{~N}^{+}$cells (white arrows, cell border). Scale bar: $5 \mu \mathrm{m}$. (B) ${ }^{2} \mathrm{H}$-glucose labeling distributions in ECs and foam cells from 3 mice. Each dot denotes an individual cell. Bars denote median \pm interquartile range. (C) ${ }^{2} \mathrm{H}$-glucose labeling distributions of nucleated foam cells as a function of whether they divided $\left({ }^{15} \mathrm{~N}^{+}\right)$during the labeling period. Data are expressed relative to the undivided $\left({ }^{15} \mathrm{~N}^{-}\right)$fraction. Nucleated intimal cells from $n=3$ mice pooled. Each dot denotes an individual cell. Bars denote mean $\pm S D$. Significance was assessed by unpaired $t$ test. (D) Mapping of ${ }^{2} \mathrm{H}$-glucose labeling in subcellular domains. Regions of interest (ROI) corresponding to individual lipid droplets (which appear black in ${ }^{12} C^{14} \mathrm{~N}^{-}$), cytosolic regions between lipid droplets, and chromatin regions $\left({ }^{31} \mathrm{P}^{-}\right.$bright) were selected using the ${ }^{12} \mathrm{C}^{14} \mathrm{~N}^{-}$and ${ }^{31} \mathrm{P}^{-}$images. ${ }^{2} \mathrm{H}$-glucose labeling data $\left({ }^{2} \mathrm{H} /{ }^{1} \mathrm{H}\right.$ ratio) was extracted for each $\mathrm{ROI}$ and is expressed in the graph (right) as relative to background. Dashed

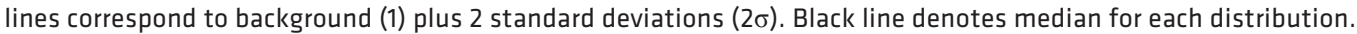
Significance was assessed by ANOVA; $P<0.0001$.

VSMCS underlying plaque are characterized by increased ${ }^{2} \mathrm{H}$-glucose labeling in the absence of augmented proliferation. We next turned our attention to glucose signal in medial VSMCs underlying the internal elastic lamina (Figure 3A, left). Surprisingly, ${ }^{2} \mathrm{H}$-glucose labeling was consistently higher in VSMCs compared with foam cells (Figure 3B). As with foam cells, VSMCs labeled with ${ }^{15} \mathrm{~N}$-thymidine had significantly higher ${ }^{2} \mathrm{H}$-glucose labeling as compared with ${ }^{15} \mathrm{~N}$-negative cells; however, the glucose labeling of medial VSMCs could not be explained by proliferation alone, as only a small subset (4\%) were concomitantly ${ }^{15} \mathrm{~N}$-labeled (Table 2 and Figure 3C). Moreover, we observed an apparent gradient of ${ }^{2} \mathrm{H}$-glucose labeling in VSMCs within the media (Figure 3A), with high levels of signal in cells underlying the center of the lesion. Indeed, quantification of glucose labeling in individual VSMCs as a function of distance from the plaque center demonstrated a significant negative correlation (Figure 3D). This finding prompted us to perform additional MIMS analyses in regions of the aorta located within the same tissue plane, but on the opposite side of the vessel wall without plaque. The VSMCs in these unaffected regions were ${ }^{15} \mathrm{~N}$-thymidine negative, and the glucose signal 
Table 1. Percent of ${ }^{15} \mathrm{~N}$-thymidine-labeled foam cells in the tunica intima ( $n=3$ mice)

\begin{tabular}{lccc}
\hline Foam cells & ${ }^{15} \mathbf{N}^{-}$ & ${ }^{15} \mathbf{N}^{+}$ & ${ }^{15} \mathbf{N}(\%)$ \\
Mouse 1 & 4 & 4 & 50 \\
Mouse 2 & 5 & 19 & 79 \\
Mouse 3 & 13 & 17 & 57 \\
Total & 22 & 40 & 65
\end{tabular}

was significantly attenuated (Figure 3E), with a 2-fold relative increase in glucose signal in the VSMCs from regions of aorta with an atherosclerotic lesion as compared with regions from the same mouse with no atheroma (Figure 3F). Overall, these data demonstrate the following: (i) lower rates of proliferation in VSMCs compared with foam cells; (ii) prominent glucose labeling in VSMCs as a function of their proximity to lesions rather than a function of proliferation; and (iii) overall higher glucose label emanating from VSMCs compared with foam cells.

Cell proliferation and glucose metabolism decrease in advanced atherosclerotic plaques. We next examined cell proliferation and glucose labeling in advanced atherosclerotic plaques. For this experiment, $L d l r^{-1-}$ mice were maintained on a $\mathrm{HF} / \mathrm{HC}$ diet for 22 weeks. In the last week, mice were again labeled with ${ }^{15} \mathrm{~N}$-thymidine and ${ }^{2} \mathrm{H}$-glucose. A modest increase in glucose label in ECs compared with foam cells was detected (Figure 4A). However, in contrast to early plaques, we detected no increase in medial VSMC ${ }^{2} \mathrm{H}$-glucose label adjacent to lesions as compared with plaque foam cells or VSMCs located in unaffected regions of aorta (Figure 4B). Coincident with this attenuation of glucose label in advanced plaques, we detected a decline in proliferative activity within the plaque. Specifically, we observed no ${ }^{15} \mathrm{~N}$-thymidine-labeled VSMCs ( $n=76$ analyzed) and a striking $85 \%$ decrease in the overall average frequency of ${ }^{15} \mathrm{~N}$-positive foam cells in lesions from individual mice (Figure 4C). These data reveal that (i) rates of foam cell proliferation in late atherosclerotic plaques were substantially reduced and (ii) glucose labeling in medial VSMCs adjacent to advanced plaques was decreased significantly out of proportion to the overall decrease in proliferation.

\section{Discussion}

Atherosclerotic lesions grow via recruitment of bone marrow-derived monocytes and local proliferation of lesional macrophages and VSMCs (14-16). This study using MIMS identified higher rates of proliferation in foam cells as compared with prior reports $(15,17)$. Differences in methods could account for these results. We measured individual ${ }^{15} \mathrm{~N}$-thymidine-labeled cells directly in plaques without the need for aortic digestion, cell sorting, or staining, which can alter the overall yield of cells. VSMCs can also dedifferentiate and express macrophage markers (18). Thus, VSMC-derived cells could contribute to the total pool of $\left({ }^{15} \mathrm{~N}^{+}\right)$foam cells identified in our study $(16,19)$. Last, the experimental approach used herein cannot distinguish whether foam cells were initially labeled locally in plaque or distally (e.g., in bone marrow). In contrast to foam cells, medial VSMC proliferation occurred in a more discrete subpopulation of cells. Whether these ${ }^{15} \mathrm{~N}$-positive VSMCs will preferentially contribute to plaque growth is the subject of ongoing work coupling lineage-tracing approaches with MIMS (14, 19). However, we did detect lipid-laden VSMCs directly underlying the intima (Figure 3), indicative of foam cell-like changes. In advanced plaques, the frequency of proliferating foam cells declined significantly. In this study, we chose to examine changes in aortic root plaque, which is an established and widely used site for assessment of atherosclerosis in mice (20). In the future, it will be important to examine other sites of lesion formation in the murine model, such as the brachiocephalic artery, given the previously described potential relevance to human plaque biology (21). Overall, our data suggest that proliferation of cells is dynamic and heterogeneous across cell types in plaques.

In dividing cells, increased energy demands heighten glucose metabolism. Here we tracked glucose at single-cell resolution directly in vivo and identified high rates of ${ }^{2} \mathrm{H}$-glucose labeling in dividing foam cells. Surprisingly, the glucose label in medial VSMCs underlying plaques exceeded that of foam cells, an effect that was lost in late plaques. These results support and extend other work demonstrating increased mRNA and protein levels of glucose transporters in medial VSMCs located in close proximity to atherosclerotic plaques in rodents and humans $(4,22)$. When considered together, these data suggest that VSMCs contribute to total glucose utilization in lesions, challenging the convention that 
A

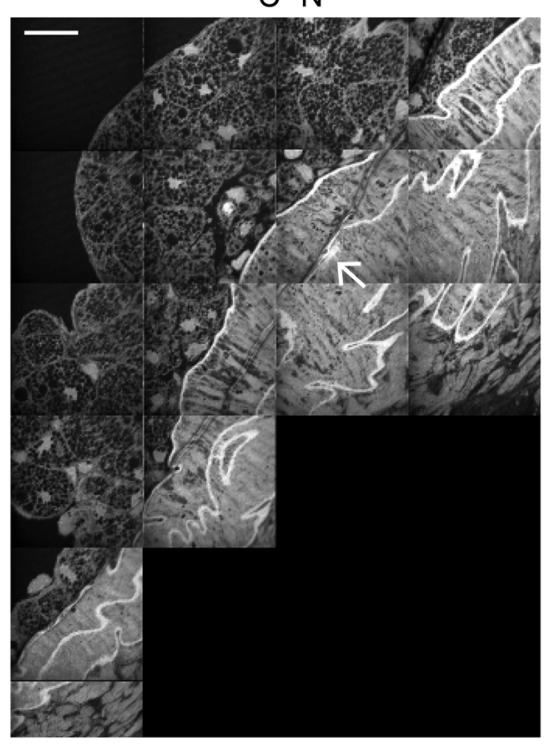

B

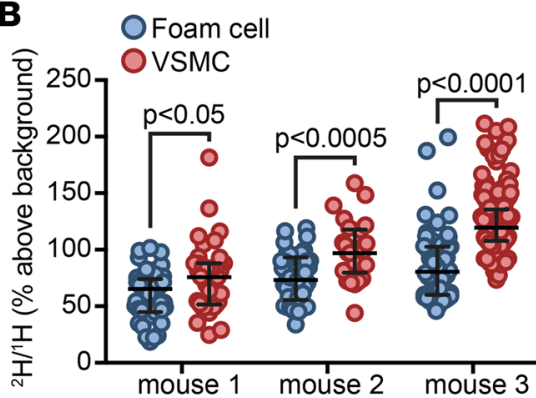

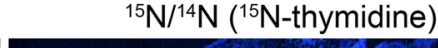

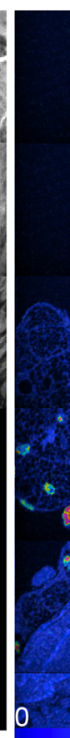

${ }^{15} \mathrm{~N} /{ }^{14} \mathrm{~N}$ ( ${ }^{15} \mathrm{~N}$-thymidine)

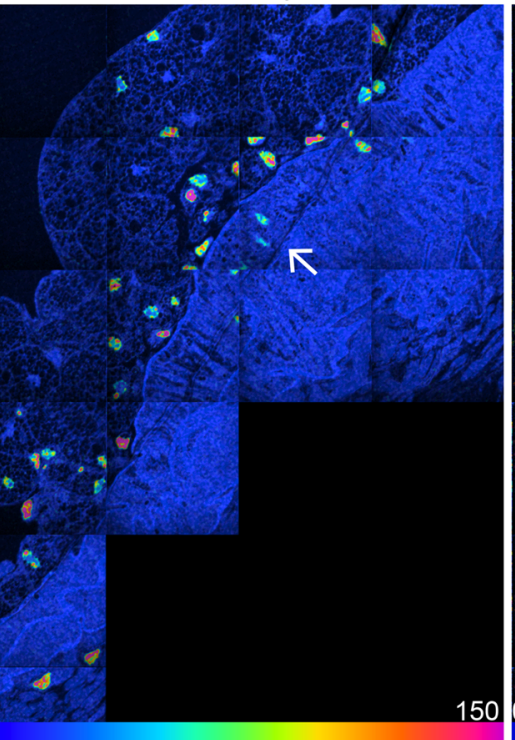

${ }^{2} \mathrm{H} /{ }^{1} \mathrm{H}$ ( ${ }^{2} \mathrm{H}-$ glucose $)$

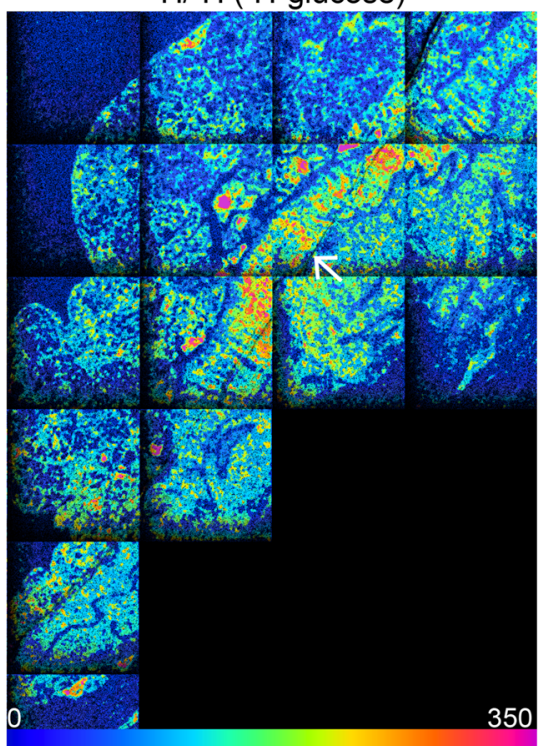

D
C

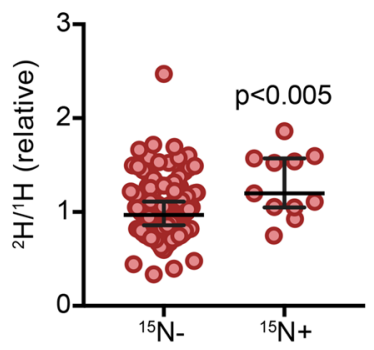

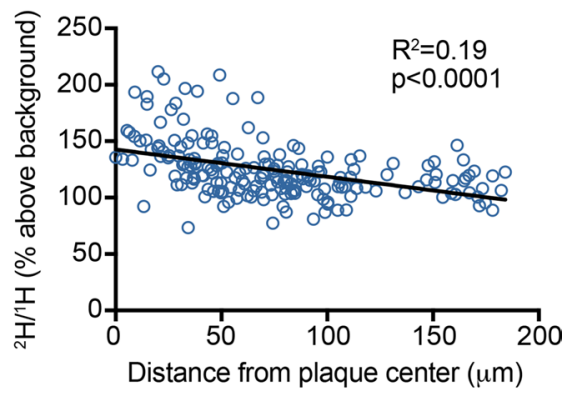

Distance from plaque center $(\mu \mathrm{m})$
E

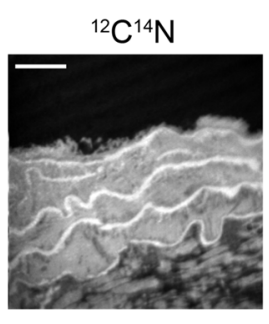

${ }^{15} \mathrm{~N} /{ }^{14} \mathrm{~N}$

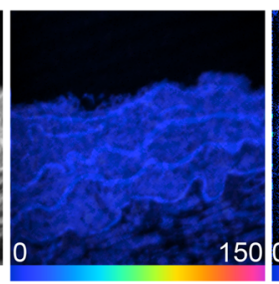

${ }^{2} \mathrm{H} /{ }^{1} \mathrm{H}$

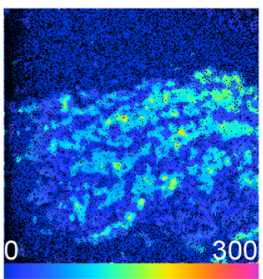

$\mathbf{F}$

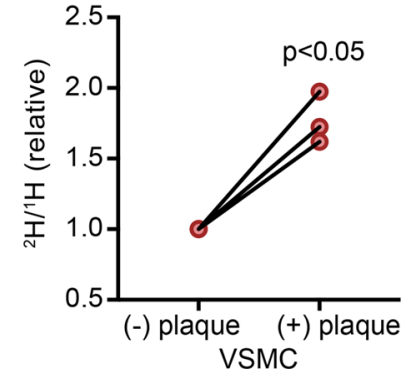

Figure 3. VSMCs underlying plaque are characterized by increased ${ }^{2} \mathrm{H}$-glucose labeling in the absence of augmented proliferation. (A) MIMS mosaic images of plaque demonstrate intense ${ }^{2} \mathrm{H}$-glucose labeling of VSMCs in the central area underneath the plaque (white arrow). Scale bar: $20 \mu \mathrm{m}$. (B) ${ }^{2} \mathrm{H}$ glucose labeling distributions of VSMCs relative to foam cells, demonstrating higher glucose utilization by VSMCs in each mouse analyzed ( $n=3$ mice). Each dot denotes an individual cell. Bars denote median \pm interquartile range. Significance assessed by Mann-Whitney $U$ test. (C) ${ }^{2} \mathrm{H}$ glucose labeling distributions of nucleated VSMCs as a function of whether they divided $\left({ }^{15} \mathrm{~N}\right.$-thymidine labeled, $\left.{ }^{15} \mathrm{~N}^{+}\right)$during the labeling period. Data expressed as relative to the undivided $\left({ }^{15} \mathrm{~N}^{-}\right)$fraction. VSMCs from $n=3$ mice were pooled. Each dot denotes an individual cell. Bars denote median \pm interquartile range. Significance was assessed by Mann-Whitney $U$ test. (D) Graph of VSMC ${ }^{2} \mathrm{H}$-glucose labeling from $\mathbf{A}$ as a function of distance from plaque center (denoted by white arrow in A). Each dot denotes an individual cell. Black line, linear regression model. (E) Representative MIMS images of regions of aortic wall without overlying visible plaque but from the same section and adjacent to regions that contained plaque. Scale bar: $10 \mu \mathrm{m}$. (F) VSMC ${ }^{2} \mathrm{H}$-glucose labeling in cells underneath plaque, relative to regions from the same section without visible plaque. Each dot represents mean VSMC labeling for the indicated region from a single mouse. $n=3$ mice. Significance was assessed by paired $t$ test.

glucose signal in plaque indicates solely inflammatory cell metabolism. One important limitation of this analysis is that we cannot exclude the possibility that ${ }^{2} \mathrm{H}$-signal reflects transfer of glucose-derived metabolites between cells. However, this phenomenon should result in a more diffuse pattern of signal across all cells. Our data demonstrate more concentrated ${ }^{2} \mathrm{H}$-signal within VSMCs, suggesting that this 
Table 2. Percentages of ${ }^{15} \mathrm{~N}$-thymidine-labeled VSMCs ( $n=3$ mice)

\begin{tabular}{lccc}
\hline VSMC & ${ }^{15} \mathbf{N}^{-}$ & ${ }^{15} \mathbf{N}^{+}$ & ${ }^{{ }^{15} \mathbf{N}^{+}}$ \\
Mouse 1 & 40 & 4 & 9 \\
Mouse 2 & 25 & 1 & 4 \\
Mouse 3 & 184 & 6 & 3 \\
Total & 249 & 11 & 4
\end{tabular}

mechanism is less plausible. These dynamic measurements of glucose labeling provide experimental evidence that metabolic heterogeneity exists between and within cell types of the growing atherosclerotic plaque in vivo.

On the basis of this first application of MIMS in atherosclerosis, we propose that several aspects of MIMS complement existing tools employed to study cell phenotypes in plaques: (i) stable isotope tracers do not alter or disrupt biochemical/metabolic pathways in the cell and thus can be administered safely over long periods of time to study rare events in animals and humans (6); (ii) labeling of cells in vivo allows direct functional assessments in the endogenous biological context during disease initiation or progression; (iii) tracers can be quantified with extreme precision within plaques without the need for additional tissue manipulation such as immunohistochemical staining or aortic digestion/cell sorting, which can alter cell viability, protein expression, or signaling; and (iv) high imaging resolution can be achieved (down to 30 $\mathrm{nm}$ laterally), which provides exquisite and quantitative detail of individual cells and subcellular compartments. In addition to these potential advantages of MIMS, certain limitations must be acknowledged. First, MIMS is expensive due to the cost of stable isotope tracers and the instrumentation. Second, image acquisition is time-consuming. This issue depends, in large part, on the abundance of an atom (or polyatomic cluster) of interest, its ionization efficiency, and the degree of effective labeling. For example, in the current study higher-throughput detection of dividing cells labeled with ${ }^{15} \mathrm{~N}$-thymidine can be achieved relative to measurement of ${ }^{2} \mathrm{H}$-glucose labeling because of an approximate order-of-magnitude difference in ion count rates, compounded by the high level and focal labeling of ${ }^{15} \mathrm{~N}$-thymidine in the nuclei of divided cells (5). Therefore, in prior work in which ${ }^{15} \mathrm{~N}$-thymidine was the only label measured, it was feasible to analyze larger swathes of tissues and/or higher numbers of mice $(5,9,10)$. As a practical matter, it will be challenging to achieve requisite statistical power to perform atherosclerosis studies comparing intergroup treatment or genetic effects without associated improvements in analytical throughput. MIMS is perhaps best suited as a method for functional studies of cellular metabolism/phenotype in which different cell populations within an animal or lesion are compared. In this case, the quantitative power of MIMS can be leveraged to achieve statistical power based on the number of measured cells, as we have demonstrated in several of our analyses herein (Figures 2 and 3). Thus, we believe MIMS offers a powerful, complementary approach to the quantitative study of cell biological processes in atherosclerosis in vivo.

In conclusion, these data reveal cellular heterogeneity of proliferation and glucose metabolism in experimental atherosclerosis. This work highlights how MIMS offers the unique capacity to perform functional measurements of cell biology in vivo at subcellular resolution. The results presented here raise important questions for future study, including defining the mechanisms regulating metabolic phenotypes of VSMCs and whether these changes are adaptive or deleterious in atherosclerosis. This study, the first to our knowledge to use MIMS in atherosclerosis, now establishes a template for follow-up mechanistic studies. In addition, the precedent set by recent first-in-human MIMS studies (12) creates an experimental framework to study plaque biology on a functional level and at single-cell resolution in humans.

\section{Methods}

Atherosclerosis model. Male Ldlr/- mice ( $n=3$ /group, The Jackson Laboratory/strain 002207) were housed in a pathogen-free facility with standard light/dark cycling and access to food and water ad libitum. An atherogenic diet (Research Diets D12108C: 40\% fat, 1.25\% cholesterol) was initiated at 8 weeks of age and continued for 9 or 22 weeks. ${ }^{15} \mathrm{~N}$-thymidine $(500 \mu \mathrm{g})$ and ${ }^{2} \mathrm{H}$-glucose $(25 \mathrm{mg})$ were obtained from Cambridge Isotope Laboratories, dissolved in sterile water, and administered by twice-daily intraperitoneal injection.

MIMS. Aortas were prepared with minor modifications (12). After perfusion with PBS (10 ml), the aortic root and distal aorta were removed and fixed in $2.5 \%$ glutaraldehyde for 10 minutes, then transferred 

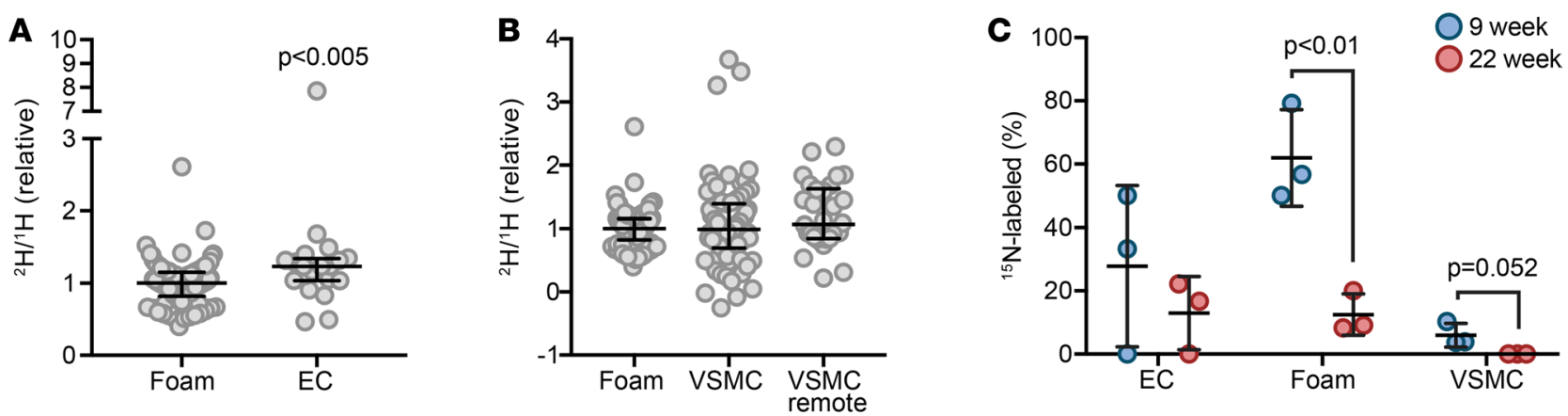

Figure 4. Cell proliferation and glucose metabolism decrease in advanced atherosclerotic plaques. (A) Intimal ${ }^{2} \mathrm{H}$-glucose labeling in advanced plaques (atherogenic diet for 22 weeks). Each dot denotes an individual cell. Data was pooled from $n=3$ mice. Bars denote median \pm interquartile range. Significance was assessed by Mann-Whitney $U$ test. (B) ${ }^{2} \mathrm{H}$-glucose labeling in foam cells versus VSMCs of advanced atherosclerotic plaques (atherogenic diet for 22 weeks). Each dot denotes an individual cell. Data were pooled from $n=3$ mice. Bars denote median \pm interquartile range. Significance was assessed by nonparametric ANOVA and Kruskal-Wallis test. (C) Frequency of proliferation $\left(\%{ }^{15} \mathrm{~N}\right.$-labeled) by cell type in established versus advanced atherosclerosis. Each dot represents the average frequency in 1 mouse ( $n=3$ mice per time point). Significance was assessed by 2-sided $t$ test.

to $4^{\circ} \mathrm{C}$ for 24 hours. In order to preserve lipid, samples were postfixed with osmium tetroxide for 12 hours, then embedded in EMbed 812 Resin (Electron Microscopy Solutions). Blocks were trimmed, oriented, and sectioned until a tissue plane was reached that contained atherosclerotic plaque as demonstrated by Toluidine blue staining (Figure 1A). Adjacent $0.5-\mu \mathrm{m}$ sections were then mounted on silicon wafers (Pure Wafer) that were custom cut to fit a 16-slot NanoSIMS sample holder. Samples were analyzed at the BWH Center for NanoImaging, using minor modifications to published parameters $(12,23)$. Silicon wafers were mounted on a NanoSIMS sample holder, then viewed by differential interference contrast microscopy (DIC). Atherosclerotic plaques identified in adjacent sections by Toluidine blue staining guided their localization by DIC and enabled recording of plaque coordinates. Samples were coated with a 15-nm layer of gold prior to NanoSIMS analysis to prevent charging, an effect often observed in resin-embedded biological samples, and that attenuates ion yield.

Samples were then analyzed with a NanoSIMS 50L instrument (CAMECA), which contains 7 detectors, enabling the measurement of 7 ionic species in parallel. In the current study, we used a previously published instrumental configuration (Supplemental Figure 2) $(12,23)$. The sample surface is sputtered with a 16-kiloelectron-volt (16-keV) cesium ion beam, enhancing the yield of negatively charged atoms and small polyatomic clusters. In a given analytical field, successive planes of increasing depth can be sputtered and analyzed until a sufficient number of ions per pixel have been counted for each ion of interest. This approach also facilitates incorporation of deflection plate peak switching, where ionic species of close mass can be acquired in an alternating manner with successively deeper analysis, thereby generating 2 different series of images. In this manner, ${ }^{15} \mathrm{~N}$-thymidine was quantified by measuring the ${ }^{12} \mathrm{C}^{15} \mathrm{~N}^{-} /{ }^{12} \mathrm{C}^{14} \mathrm{~N}^{-}$ratio $(5,10)$ and ${ }^{2} \mathrm{H}$-glucose was quantified by measuring the ${ }^{12} \mathrm{C}_{2}{ }^{2} \mathrm{H}^{-} /{ }^{12} \mathrm{C}_{2}{ }^{1} \mathrm{H}^{-}$ratio in alternating fashion within each analytical

\section{Table 3. Key resources}

\begin{tabular}{|c|c|c|}
\hline Reagent or resource & Source & Identifier \\
\hline \multicolumn{3}{|l|}{ Chemicals } \\
\hline${ }^{15} \mathrm{~N}$-thymidine & Cambridge Isotope Laboratories & NLM-3901-PK \\
\hline${ }^{2} \mathrm{H}$-glucose & Cambridge Isotope Laboratories & CLM-1396-1 \\
\hline $25 \%$ Glutaraldehyde solution & Electron Microscopy Solutions & Catalog 16220 \\
\hline $4 \%$ Osmium tetroxide solution & Electron Microscopy Solutions & Catalog 18459 \\
\hline EMbed 812 Resin & Electron Microscopy Solutions & Catalog 14120 \\
\hline \multicolumn{3}{|l|}{ Experimental models, organisms/strains } \\
\hline Mouse: B6.129S7-Ldlrtm1Her/J (Ldlr KO) & The Jackson Laboratory & Stock no. 002207 \\
\hline \multicolumn{3}{|l|}{ Other } \\
\hline $40 \%$ Fat, $1.25 \%$ cholesterol diet, irradiated & Research Diets & Catalog D12108Ci \\
\hline
\end{tabular}


field $(12,23)$. In order to analyze the full area of each atherosclerotic plaque, we performed automated chain analysis, wherein adjacent $50 \mu \mathrm{m} \times 50 \mu \mathrm{m}$ fields were analyzed and then tiled together. Because such images were derived from analysis of the uppermost atomic layers of the $0.5-\mu \mathrm{m}$-thick section, features of interest could be reanalyzed at higher lateral resolution by sputtering away additional layers with a smaller cesium ion probe. Image files were visualized and analyzed with a custom plugin to ImageJ:OpenMIMS 3.0: https:// github.com/BWHCNI/OpenMIMS. Isotope ratio data were shown as hue saturation intensity (HSI) images. The lower bound of the scale (blue) was set at natural background as verified by analysis of unlabeled samples and/or embedding resin (e.g., for ${ }^{15} \mathrm{~N}$-data a lower bound of 0 is equivalent to the natural background of $0.37 \%$ and an upper bound of 100 would correspond to a ratio of $0.74 \%$ ). The upper bound of the scale was set to demonstrate regional differences in enrichment; any changes to the scale will affect the color pattern and thus the visual interpretation of the data, but the underlying quantitative data remain unmodified. Table 3 provides a list of key reagents and catalog numbers.

Statistics. Statistical analyses were performed with Prism. Normality was assessed using a Shapiro-Wilk test. For comparisons of 2 normal distributions, we performed a 2-sided $t$ test; for nonparametric data, we performed a Mann-Whitney $U$ test. For comparisons of 3 or more groups in which at least 1 group was non-normally distributed, we performed ANOVA and Kruskal-Wallis test.

Study approval. Animal experiments were conducted at Vanderbilt University Medical Center (VUMC) as approved by the Institutional Animal Care and Use Committee (Vanderbilt Office of Animal Welfare Assurance) and conducted in accordance with the NIH Guide for the Care and Use of Laboratory Animals (National Academies Press, 2011).

\section{Author contributions}

CG, MFL, MLS, and JDB designed experiments. SPD dosed animals with isotope tracers and harvested tissue for mouse experiments. CG performed NanoSIMS analysis. AGW and VRB assisted with sample preparation. CG, AGW, MLS, and JDB analyzed data. MLS and JDB prepared figures and wrote the manuscript.

\section{Acknowledgments}

We acknowledge support from NIH research grants HL116263, HL127173, DK59637 (MFL) and the Vanderbilt Young Ambassadors Award (JDB). We wish to thank Maria Vinogradova at the VUMC electron microscopy core for help with tissue sectioning and embedding. We thank the Atherosclerosis Research Unit core laboratory for help with harvesting aortas. Also, we thank Joshua Beckman for helpful discussions during manuscript preparation.

Address correspondence to: Jonathan D. Brown, VUMC, 2220 Pierce Ave, Preston Building 352, Nashville, Tennessee 37232, USA. Phone: 617.686.9918; Email: jonathan.d.brown@vumc.org.

1. Libby P, et al. Inflammation, immunity, and infection in atherothrombosis: JACC review topic of the week. J Am Coll Cardiol. 2081;72(17):2071-2081.

2. Koelwyn GJ, Corr EM, Erbay E, Moore KJ. Regulation of macrophage immunometabolism in atherosclerosis. Nat Immunol. 2018;19(6):526-537.

3. Andrejeva G, Rathmell JC. Similarities and distinctions of cancer and immune metabolism in inflammation and tumors. Cell Metab. 2017;26(1):49-70.

4. Folco EJ, et al. Hypoxia but not inflammation augments glucose uptake in human macrophages: implications for imaging ath erosclerosis with 18fluorine-labeled 2-deoxy-D-glucose positron emission tomography. J Am Coll Cardiol. 2011;58(6):603-614.

5. Steinhauser ML, et al. Multi-isotope imaging mass spectrometry quantifies stem cell division and metabolism. Nature. 2012;481(7382):516-519.

6. Steinhauser ML, Lechene CP. Quantitative imaging of subcellular metabolism with stable isotopes and multi-isotope imaging mass spectrometry. Semin Cell Dev Biol. 2013;24(8-9):661-667.

7. Zhang DS, et al. Multi-isotope imaging mass spectrometry reveals slow protein turnover in hair-cell stereocilia. Nature. 2012;481(7382):520-524.

8. He C, et al. NanoSIMS analysis of intravascular lipolysis lipid movement across capillaries into cardiomyocytes. Cell Metab. 2018;27(5):1055-1066.e3.

9. Senyo SE, et al. Mammalian heart renewal by pre-existing cardiomyocytes. Nature. 2013;493(7432):433-436.

10. Kim SM, et al. Loss of white adipose hyperplastic potential is associated with enhanced susceptibility to insulin resistance. Cell Metab. 2014;20(6):1049-1058.

11. Lechene $\mathrm{C}$, et al. High-resolution quantitative imaging of mammalian and bacterial cells using stable isotope mass spectrometry J Biol. 2006;5(6):20. 
12. Guillermier C, et al. Imaging mass spectrometry demonstrates age-related decline in human adipose plasticity. JCI Insight. 2017;2(5):e90349.

13. Guillermier C, Steinhauser ML, Lechene CP. Quasi-simultaneous acquisition of nine secondary ions with seven detectors on NanoSIMS50L: application to biological samples. Surf Interface Anal. 2014;46(suppl 1):150-153.

14. Misra A, et al. Integrin beta3 regulates clonality fate of smooth muscle-derived atherosclerotic plaque cells. Nat Commun. 2018;9(1):2073.

15. Robbins CS, et al. Local proliferation dominates lesional macrophage accumulation in atherosclerosis. Nat Med. 2013;19(9):1166-1172.

16. Shankman LS, et al. KLF4-dependent phenotypic modulation of smooth muscle cells has a key role in atherosclerotic plaque pathogenesis. Nat Med. 2015;21(6):628-637.

17. Lindau A, et al. Atheroprotection through SYK inhibition fails in established disease when local macrophage proliferation dominates lesion progression. Basic Res Cardiol. 2016;111(2):20.

18. Rong JX, Shapiro M, Trogan E, Fisher EA. Transdifferentiation of mouse aortic smooth muscle cells to a macrophage-like state after cholesterol loading. Proc Natl Acad Sci US A. 2003;100(23):13531-13536.

19. Chappell J, et a. Extensive proliferation of a subset of differentiated, yet plastic, medial vascular smooth muscle cells contributes to neointimal formation in mouse injury and atherosclerosis models. Circ Res. 2016;119(12):1313-1323.

20. Daugherty A, et al. Recommendation on design, execution, and reporting of animal atherosclerosis studies: a scientific statement from the American Heart Association. Arterioscler Thromb Vasc Biol. 2017;37(9):e131-e157.

21. Rosenfeld ME, Polinsky P, Virmani R, Kauser K, Rubanyi G, Schwartz SM. Advanced atherosclerotic lesions in the innominate artery of the ApoE knockout mouse. Arterioscler Thromb Vasc Biol. 2000;20(12):2587-2592.

22. Wall VZ, et al. Smooth muscle glucose metabolism promotes monocyte recruitment and atherosclerosis in a mouse model of metabolic syndrome. JCI Insight. 2018;3(11):e96544.

23. Guillermier C, Poczatek JC, Taylor WR, Steinhauser ML. Quantitative imaging of deuterated metabolic tracers in biological tissues with nanoscale secondary ion mass spectrometry. Int J Mass Spectrom. 2017;422:42-50. 